Too often articles and papers, especially

research papers and scientific studies, seem to

go out of their way to make their writing as

difficult as possible... Yet surely the essence of

good writing is to communicate and inform

and especially to be understood?

\section{Readable writing}

The Comment on page 694 of this edition of the BDJ (commenting on the paper Staphylococcal aureus infection in the oral cavity: a 3-year retrospective analysis of clinical laboratory data) contains a phrase in the final sentence that I have never seen before attributed to a scientific research paper in the $B D J$ - or any other scientific journal for that matter. The comment is quite simple. It states 'The paper provides a very readable account...' That is all.

To me this is an extremely powerful statement. Too often articles and papers, especially research papers and scientific studies, seem to go out of their way to make their writing as difficult to read as possible. This is so common that it has become both expected and encouraged. Yet surely the essence of good writing is to communicate, to inform and/or persuade, and especially to be understood? Should that have been a rhetorical question? Are there people who believe that good writing should confuse, mislead, misinform and obfuscate? Apart from the world of propaganda and some copy-writing, I do not think so.

Yet struggling through many of the scientific papers published in the $B D J$ can often be difficult for readers, whether they are scientists or not. It is almost as if the writer feels a need to write in what is often termed 'scientific-eeze', adopting a style of writing that uses complex structures, very long sentences and long words when shorter ones will do (my own pet hate is the word 'utilise' instead of 'use').

I have often pondered on the reason for this. I have been told that academics are trained into this style of writing and that their career can depend on being able to write 'appropriately'. I know that often people writing a thesis or dissertation are advised to write in 'scientificeeze' if they want to pass. I have also been told (by a prominent academic at one of the UK dental schools) that he would not dream of submitting a paper to the $B D J$ if the other papers were written too simply. The problem was he did not want his paper to be seen among other papers that were too easy to read (and thus by his implication a lower standard of scientific quality). I must admit this last comment left me astonished at the time. It was very early in my role as editor of the $B D J$ and I was still fairly naive about scientific publishing.

What is even more perplexing is that scientists themselves admit to preferring to read papers with a simpler, easier, understandable style; yet when it comes to writing, they seem to forget what they like to read. They lapse into sentences such as 'From the evidence of this study it is clear that using guidelines as a yardstick of success, present methods of updating education and training are largely ineffective as a way of improving cross-infection control yet at the same time respondents are eager to improve their practice because $75 \%$ of the population completed the questionnaire and nearly twothirds of them wanted to know more about crossinfection control procedures' Surely the author could have put that more simply?

Whatever the reason, scientific writing tends to be difficult to read, which is why I found the comment on the paper in this issue so unusual. I must confess that eleven years of proof-reading papers has built a kind of automatic ability for me to be able to absorb them, but I still yearn for the refreshing change of a really wellwritten paper - and they do exist.

Perhaps they are so few because, paradoxically, it is much harder to write something easy to read than something that is not. One reason for this is that people often do not write what they really mean, and if they omit the editing stage of writing (this is the stage when you go back through your writing to edit it yourself) then they do not appreciate the difference between what they meant to write - and what they have written. Another reason for complex writing could be the fact that often people feel there should be a difference between what is said and what is written. This may be strictly true, but reading your writing out loud often reveals the complexity and confusion only too well.

Complex writing in scientific publishing remains a mystery. Why does it continue to hold sway when all the editors I meet abhor it and long for authors who write simply? Now there is a question.

Mike Grace, Editor m.grace@bda.org doi:10.1038/sj.bdj.4810818 